\title{
Lasers with nonlocal feedback, diffraction, and diffusion
}

\author{
Francesco Papoff \\ Department of Physics, University of Strathclyde, 107 Rottenrow, Glasgow G4 0NG, United Kingdom \\ Roberta Zambrini \\ IFISC (CSIC-UIB), Campus Universitat Illes Balears, 07122 Palma de Mallorca, Spain
}

(Received 27 December 2008; published 9 March 2009)

\begin{abstract}
An off-axis feedback loop originates a two-point nonlocality that changes the instabilities of lasers of classes A and B: after crossing a first threshold these devices amplify while they lase above a second one. We obtain the instability diagram in presence of off-axis feedback and show that transverse phase and group velocities can be tuned to have the same or opposite sign, depending on control parameters. Counterpropagating noise sustained patterns and localized perturbations are found for an out-of-phase feedback when the laser operates as a signal splitter. We predict these effects in a broad class of devices, with either positive or negative refractive index, diffusion, and in presence of noise.
\end{abstract}

DOI: 10.1103/PhysRevA.79.033811

PACS number(s): 42.65.Sf, 05.40.Ca, 42.60.Da, 05.45.-a

\section{INTRODUCTION}

The importance of feedback loops in dynamical systems has been long recognized not only in different fields of physics but also in biology and engineering [1,2]. In the context of optics, the combination of media nonlinearity and feedback is at the basis of spatiotemporal instabilities so fundamental as laser emission $[3,4]$. Recently, a great attention has been devoted to the role of temporal delay on the spectral, dynamical, and stochastic properties of the light emitted by nonlinear devices with external feedback, also in view of applications $[5,6]$. In this paper we consider the effects introduced by off-axis external feedback on a broad class of resonators containing amplifiers. The off-axis feedback is modeled by a two-point nonlocality that is the spatial analogous of temporal delay. The presence of tilted or off-axis beams is a common experimental issue and has also been subject of both theoretical and experimental studies in liquid crystals light valves [7-9], Kerr-type media [10-12], and generic nonlinear systems with diffusive [13] and diffractive [14] couplings.

In this paper we examine in detail the effect of off-axis feedback in a broad class of devices containing active media with fast decay of the polarization [15], including media with negative refraction index [16] and devices with soft apertures [17]. We show how an off-axis feedback changes the nature of the first instability threshold, which without offset is a lasing threshold. As a consequence, there are large windows of control parameters where small localized signals can be strongly amplified while the background radiation in other region of the system remains very low [14]. In Sec. II we give a thorough explanation of the theoretical method used to calculate the instability diagram, first reported in Ref. [14], showing by some examples the complexity of the analysis. Mathematical details of these calculations are summarized in Appendix A. The roles of two-point nonlocality, diffusion, diffraction, and feedback on instability threshold are taken into account. Furthermore, the signal moves across the cavity with transverse phase and group velocities that are easily managed to have the same or opposite signs [14]. In spite of the broken transverse reflection symmetry, localized pertur- bations can move both toward or against the offset direction and can even split into two counterpropagating components, with the laser operating as a signal splitter. Several numerical simulations of the full nonlinear model confirm our theoretical analysis: peculiar noise sustained patterns are shown in Sec. III, while in Sec. IV we consider the spatiotemporal evolution of localized perturbation. We show that the operation of the signal splitter persists in presence of added noise, which is very important from the point of view of experiments. We consider mainly systems in which the time delay of the feedback is negligible with respect to the time scale of the field amplitude, but, as discussed in Appendix B, the phenomena reported in this paper are present also when the time delay of the feedback cannot be neglected.

\section{NONLOCALITY IN OPTICAL SYSTEMS WITH DIFFRACTION AND DIFFUSION}

We consider class A systems described by nondimensional equations of the type

$$
\partial_{t} E=f\left(|E|^{2} ; \mu\right) E+\left(d_{R}+i d_{I}\right) \partial_{x x}^{2} E+r e^{i \theta} E_{\Delta x},
$$

and class $\mathrm{B}$ systems described by

$$
\begin{gathered}
\partial_{t} E=g_{1}\left(|E|^{2}, N ; \mu\right) E+\left(d_{R}+i d_{I}\right) \partial_{x x}^{2} E+r e^{i \theta} E_{\Delta x}, \\
\partial_{t} N=g_{2}\left(|E|^{2}, N, \partial_{x x}^{2} N ; \mu\right),
\end{gathered}
$$

where $E$ is the slowly varying amplitude of the electric field, $N$ is the population inversion, $d_{R}, d_{I}$ are the diffusion and diffraction coefficients, ${ }^{1}$ and $\mu$ is a control parameter. The nonlocal coupling of the field $E$ at time $t$ in a point $x$ with the field $E_{\Delta x}$ at time $t-\Delta t$ in a point $x+\Delta x$ is the consequence of an off-axis single-passage feedback loop. We consider feedback loops for which the time delay $\Delta t$ is very small compared to the time scale of the slowly varying envelope of the field; the feedback can then be characterized by an amplitude

\footnotetext{
${ }^{1}$ Notation: the subscripts $R$ and $I$ are used to distinguish real and imaginary parts of complex variables in the rest of the paper.
} 


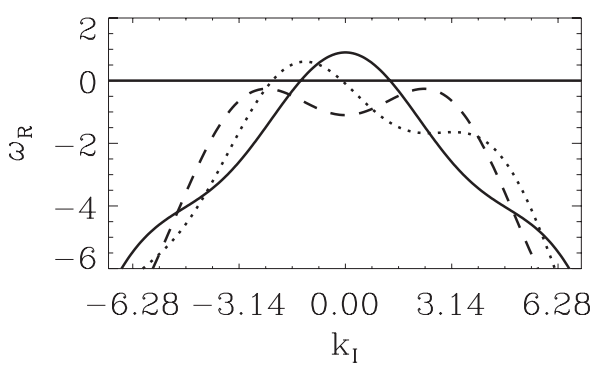

FIG. 1. Dispersion relation $\omega_{R}$ for $\beta_{R}=-0.1, r=1, \Delta x=0.9$, and $\delta=0.45 \pi$. The effect of the feedback phase $\theta$ is shown: for $\theta=0$ (continuous line) the most unstable mode is the homogeneous one, for $\theta=\pi / 2$ (dotted line) a phase pattern is predicted, and for $\theta=\pi$ (dashed line) two opposite wave numbers have the same real dispersion. The size of the oscillations in the $\omega_{R}(k)$ function increases with $r$.

$0<r<1$ and a phase shift $\theta=\omega_{L} \Delta t$, where $\omega_{L}$ is the carrier frequency. $f, g_{1}$, and $g_{2}$ are nonlinear complex functions that can be derived with respect to $E$ and $N$.

Equation (1) and Eqs. (2) and (3) have trivial solutions $E=0$ and $E=N=0$, respectively. Note that the following analysis actually applies to a more general class of equations in which the feedback term is $\left[f_{1}\left(|E|^{2}\right) E\right]_{\Delta x}$, with $r e^{i \theta}=f_{1}(0)$.

The dispersion relation for perturbations $\delta E \sim \exp (\omega t$ $\left.+i k_{I} x\right)$ is

$$
\omega=\beta-e^{i \delta} k_{I}^{2}+r e^{i\left(\theta+k_{I} \Delta x\right)},
$$

where we have used the exponential notation $d_{R}+i d_{I}=|d| e^{i \delta}$ and rescaled $x$ in units of $|d|$. Here $\beta=f(0)$ for Eq. (1) and $\beta=g_{1}(0,0)$ for Eq. (2). For class B models, perturbations $\delta E$ and $\delta N$ of $E=N=0$ are decoupled [this is given by the condition $\left.\partial_{N} g_{1}(0,0)=\partial_{E} g_{2}(0,0)=0\right]$ and perturbations $\delta N$ are always damped and can be ignored. From Eq. (4) we can find that there are bands of $k_{I}$ which are unstable (Fig. 1): the most unstable $k_{I}$ are given by

$$
\begin{aligned}
& \partial_{k_{I}} \omega_{R}=-\left[2 k_{I} \cos \delta+r \Delta x \sin \left(k_{I} \Delta x+\theta\right)\right]=0, \\
& \partial_{k_{I}^{2}}^{2} \omega_{R}=-\left[2 \cos \delta+r \Delta x^{2} \cos \left(k_{I} \Delta x+\theta\right)\right]<0 .
\end{aligned}
$$

Moreover, we see that the instability threshold can be expressed as a function of four relevant parameters, namely, $\phi, \delta, r \Delta x^{2}$, and $\beta_{R} \Delta x^{2}$; therefore increasing the shift size $\Delta x$ produces on the device the same effect of larger gain $\beta_{R}$ and feedback $r$. A specific effect of the nonlocality is that the relative strength of diffusion and diffraction, $\delta$, also becomes an effective parameter to control the threshold position. As a matter of fact, the lowest gain and feedback thresholds are generally found in the purely diffractive limit $(\delta \sim \pi / 2)$. The effect of diffusion on the feedback lasing threshold is as follows: for any not vanishing feedback phase $\phi$, the threshold value for the scaled feedback strength $r \Delta x^{2}$ increases with the diffusion, being independent of the sign of the refractive index ( $\operatorname{sign}$ of $\delta$ ). Both $\beta_{R}$ and $r$ can be increased to cross the laser threshold and-similarly to the case of perfect alignment - if the feedback is out of phase then stronger gain is required. For fixed values either of the gain or of the

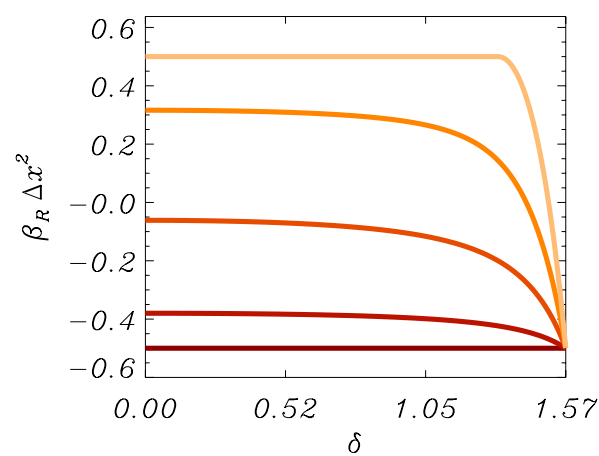

FIG. 2. (Color online) Instability thresholds for $r \Delta x^{2}=0.5$ and for $\phi=n \pi / 4$ with $n=0,1,2,3,4$ (from bottom to top). The lowest threshold is found for $\phi=0$ and the instability takes place above the lines.

feedback the nonlocality strongly decreases the threshold values for the gain as well as for the feedback field. The fact that a misalignment lowers the threshold is rather surprising: it is due to the fact that the most unstable mode has $k_{I} \neq 0$ so that the effect of the nonlocal coupling is actually equivalent to a reduction in the feedback dephasing. Consistently with this interpretation, in the case of feedback perfectly in phase with the intracavity field $(\phi=0)$ the threshold is independent of the lateral shift $\Delta x$ because the most unstable mode is the homogeneous one $\left(k_{I}=0\right)$. Instability diagrams for different control parameters have been reported in Ref. [14]. In Fig. 2 we show that the dependence of the threshold position on the relative size of diffusion and diffraction is rather slow (Fig. 2) if there is a significantly large diffusion. A fast change in the threshold values is found, however, when the purely diffractive case is approached $(\delta \rightarrow \pi / 2)$. The feedback detuning $\phi$ increases the threshold value for the pump (here shown) as well as for the feedback $(r)$.

The dispersion Eq. (4) has in general a not vanishing imaginary part. The phase velocity is

$$
v_{p}=-\frac{\omega_{I}\left(k_{I}\right)}{k_{I}}=k_{I} \sin \delta-\frac{\beta_{I}+r \sin \left(k_{I} \Delta x+\theta\right)}{k_{I}},
$$

which for the most unstable wave number $k_{I_{c}}$

$$
v_{p}\left(k_{I_{c}}\right)=k_{I_{c}} \sin \delta-\frac{\beta_{I}}{k_{I_{c}}}+\frac{2 \cos \delta}{\Delta x} .
$$

The group velocity is

$$
\begin{aligned}
v_{g} & =2 k_{I_{c}} \sin \delta-r \Delta x \cos \left(k_{I_{c}} \Delta x+\theta\right) \\
& =2 k_{I_{c}} \sin \delta \mp \sqrt{1-\frac{4 k_{I_{c}}^{2} \cos \delta^{2}}{r^{2} \Delta x^{2}}},
\end{aligned}
$$

where the - sign always satisfies Eq. (6) and therefore corresponds to a wave packet with frequency spectrum centered on a local maximum of the amplification. On the contrary, the + sign may not satisfy Eq. (6). From Eq. (9) we can see that the group velocity is null for

$$
k_{I_{c}}=r \Delta x / 2,
$$




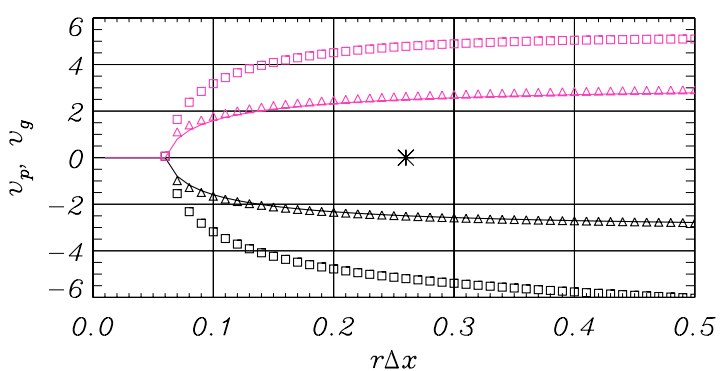

FIG. 3. (Color online) Phase (triangles) and group (squares) velocities for $\theta=\pi, \delta=0.49 \pi$, and $\beta=-0.02-0.2$. The upper curves are the velocities for $k_{c}$ (continuous line) and the lower (black) curves refer to $-k_{c}$. For $r \Delta x>0.26$ (see the star) the homogeneous state is unstable.

$$
\frac{r \Delta x^{2}}{2}=-(\theta+\delta)+\left(2 n+\frac{1}{2}\right) \pi
$$

and

$$
\begin{gathered}
k_{I_{c}}=-r \Delta x / 2, \\
\frac{r \Delta x^{2}}{2}=\theta-\delta-\left(2 n+\frac{1}{2}\right) \pi .
\end{gathered}
$$

When Eq. (6) is satisfied, these manifolds in the control parameter space separate regions in which the group and the phase velocity have the same sign from region in which these velocities have opposite sign. In particular, there are manifolds in which $v_{p}\left(k_{I_{c}}\right)$ is null, which separate regions of positive and negative phase velocities. From the existence of these manifolds, we deduce that in these systems there are regions of the control parameters where the phase and group velocities have the same sign and region where they have opposite sign. Moreover, the real part of the dispersion relation may have more than one maximum so that a single perturbation may split into two wave packets. This situation is predicted, for instance, in Fig. 3, representing the phase and group velocities of wave packets of critical wave numbers with opposite signs. In the purely diffractive limit $\delta \rightarrow \pi / 2$, both velocities are actually odd functions of $k_{c}$; this symmetry is reduced by the effect of diffusion $(|\delta|<\pi / 2)$. Therefore, even if for $\phi=\pi$ both $+k_{c}$ and $-k_{c}$ are unstable, from the linear analysis we do not expect intensity stripe patterns above threshold.

Note that the tunability of transverse phase and group velocities is a general property that is valid also in the case in which $\Delta t$ is of the order of the time scale of the slowly varying amplitude, as shown in Appendix B. This tunability is therefore a rather robust and distinctive feature of twopoint nonlocality with respect to models where velocities are induced by drift terms [18].

When perturbations have non-null group velocities, one needs to consider whether the perturbations grow fast enough to occupy the entire system (absolute instability) or if their group velocity is such that the perturbations, although growing, eventually move away and disappear (convective instability). Note that perturbations may be transiently am-

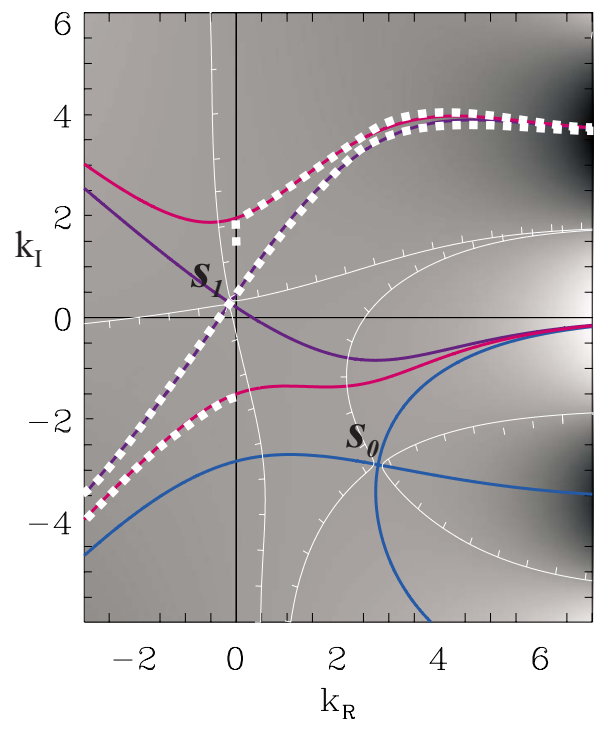

FIG. 4. (Color online) Dispersion relation in the $k_{R}, k_{I}$ plane. Equiphase lines (in colors) and equiamplitude lines (white with ticks). Ticks point in the direction of decreasing dispersion in order to identify the steepest character of the equiphase lines. The equiphase lines that are part of the rather peculiar integration path are marked with a thick dotted line. The correct evaluation of the absolute threshold comes disregarding the saddle point $s_{0}$ as it is not part of the integration path. Parameters: $\Delta x=0.875, r=0.8, \theta=0$, and $\delta=0.45 \pi$. Here $k_{I_{m}} \sim-1.36$ and $k_{I_{M}} \sim 1.36$.

plified before disappearing also in systems where the linear stability operator is non-normal, i.e., does not commute with its adjoint [19]. This type of transient growth, however, manifests itself when the state is stable, while convective instability happens, of course, when the state loses its stability. In particular, in the systems considered here the linear stability operator does commute with its adjoint so there is no transient growth, but there is convective instability.

In order to calculate absolute thresholds, we need to analyze the dispersion relation and evaluate asymptotically the Green's function by analytically extending the dispersion relation to complex wave vectors $k$ and finding the appropriate integration paths in the plane $k$. A detailed analysis is given in Appendix A, here we remark only that the asymptotic evaluation of the Green's function cannot be done by closing the integration contour using only equiphase lines from saddle points, as in the purely diffusive case. However, only the part of the imaginary axis $k_{I}$ with $w_{R}>0$ contributes asymptotically to the Green's function. Therefore, we only need to close with steepest-descent paths a finite segment $\left[k_{I_{m}}, k_{I_{M}}\right]$ with $k_{I_{m}}$ and $k_{I_{M}}$, such that $w_{R}\left(0, k_{I}\right)<0$ for $k_{I}$ $\leq k_{I_{m}}$ and $k_{I} \geq k_{I_{M}}$. The correct determination of the absolute thresholds requires to identify the integration paths and to evaluate the contribution to the Green's functions of the saddle points that are part of it, excluding all the others. For instance, the saddle $s_{1}$ must be included in the integration path in Fig. 4 and the saddle $s_{0}$ must be excluded; the opposite happens in Fig. 5. We point out that the saddles outside the integration path can have real dispersion much larger than that of the saddles on the path; therefore, their erroneous inclusions would give an instability diagram drastically different from the correct one. 


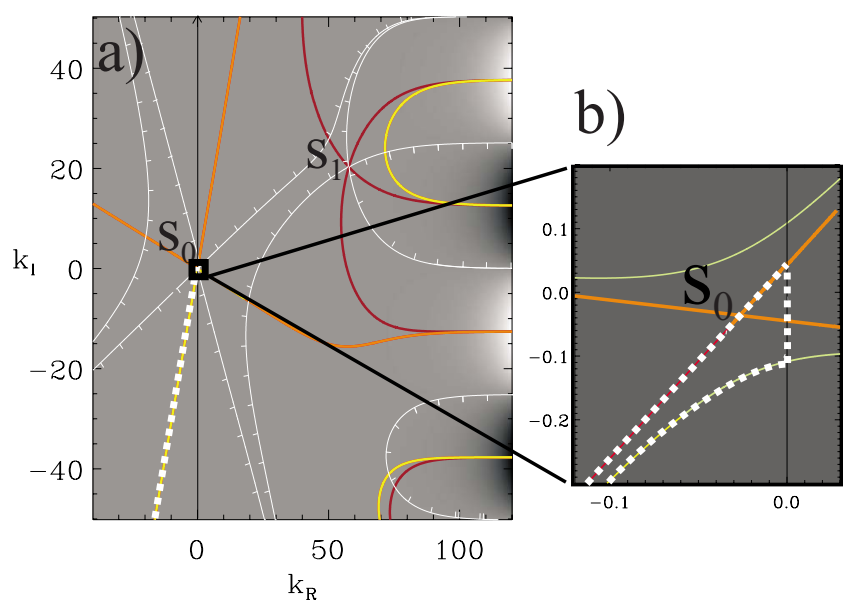

FIG. 5. (Color online) Dispersion relation notation as above. The integration path includes only one saddle, $s_{0}$, and is composed by a steepest descent passing from $s_{0}$, another equiphase line, and a segment of the imaginary axis. The saddle point $s_{1}$, with huge dispersion $w_{R}\left(k_{1}\right)$, needs to be discarded because it does not belong to the integration path. Parameters: $\Delta x=0.125, r=0.72, \theta=\pi / 2$, and $\delta=0.2 \pi$. Here $k_{I_{m}} \sim-0.1$ and $k_{I_{M}} \sim 0$.

The absolute threshold dependence on $\Delta x$ is smooth for small feedback $r$ [Fig. 7(b)], while a not monotonic behavior is found increasing the feedback strength. In the case shown in Fig. 7(a) $(r=0.8)$, for instance, this happens for shift $\Delta x$ $\sim 2.5$, where a sudden lowering of the threshold appears. This sudden variation is due to a change in the "leading" saddle point, i.e., the one with larger real dispersion. Looking at Fig. 6, we can see that in this case the integration path includes two saddles, $s_{1}$ and $s_{2}$. The sudden variation in the threshold happens when the shift decreases and $s_{1}$ does not belong anymore to the integration path.

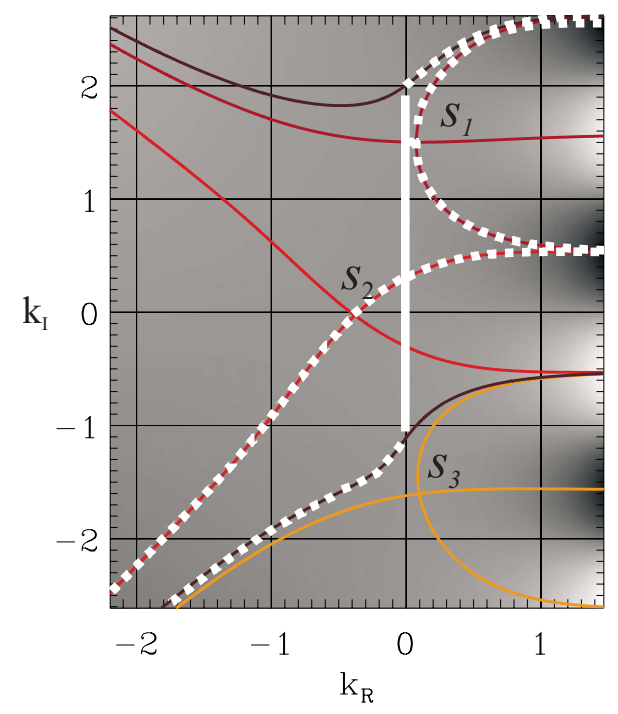

FIG. 6. (Color online) Same notation as above. The two saddles $s_{1,2}$ have positive real dispersion with $\omega_{R}\left(s_{1}\right)>\omega_{R}\left(s_{2}\right)$, while $\left(s_{3}\right)$ has negative dispersion and does not belong to the integration path. Decreasing the shift from $\Delta x=3$ to $\Delta x=2$, the contour path can be closed considering just the saddle $s_{2}$ even if the dispersion in $s_{1}$ is larger. Parameters: $\Delta x=3, r=0.8, \theta=\pi / 2$, and $\delta=0.45 \pi$. Here $k_{I_{m}}$ $\sim-1$ and $k_{I_{M}} \sim 1.8$.
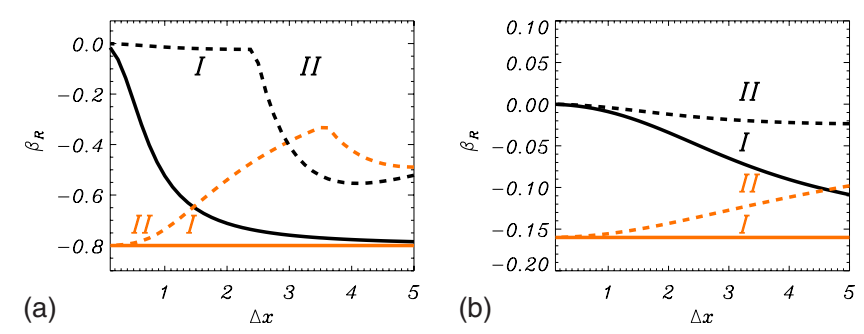

FIG. 7. (Color online) Instability thresholds for (a) $\delta=0.45 \pi$, $r=0.8$ and (b) $\delta=\pi / 4, r=0.16$. The convectively unstable region (i) is comprised between the continuous and dashed lines. The system is absolutely unstable above the dashed lines. The colored curves are found for $\phi=0$ and the black ones for $\phi=\pi / 2$.

\section{SPONTANEOUS PATTERNS IN LASER A EQUATION}

The stability analysis as well as the study of phase and group velocities are used in the present and the following sections to understand the main characteristics of the spatiotemporal dynamics of patterns as well as localized light spots of a full nonlinear model, the class A laser:

$$
\frac{\partial}{\partial t} E=-E(1+i \theta-N)+e^{i \delta} \nabla^{2} E+r e^{i \phi} E(x+\Delta x)+\epsilon \xi(x, t),
$$

$$
N=\frac{\mu}{1+|E|^{2}},
$$

with $\xi(x, t)$ Gaussian white noise. Numerical simulation of this model, ${ }^{2}$ exploring different parameter regions and boundary conditions agrees with our predictions for both absolute and convective instability thresholds. In particular, this model reproduces the sudden change in the threshold with the shift when increasing the feedback shown in Fig. 7(a). This confirms indeed the importance as well as the validity of an analysis that for systems with nonlocal couplings is rather demanding.

Laser (14) in the convectively unstable regime displays incoherent patterns that are the result of macroscopic amplification of noise [20]. Wavelengths and velocities are in good agreement with Eqs. (5), (8), and (9), showing that these characteristics of the amplified traveling waves can be predicted by a linear analysis. As an example, the dominant pattern shown in Fig. 8 has a wave vector $\sim-0.46$ in good agreement with the maximum of the dispersion relation $k_{I}=-0.459$. The phase velocity of this pattern is $v_{\mathrm{ph}} \sim-0.6$ [see real part of the field in Fig. 8(b)] in agreement with the analytical value -0.576 . The group velocity can be approximately estimated also looking at traveling fronts and wave packets in spatiotemporal diagrams of the intensity of the field [Fig. 8(b)]. The numerically estimated value $\sim-0.95$ and the analytical one -0.977 are again in reasonable agreement.

\footnotetext{
${ }^{2}$ Numerical simulations with a second-order in time Runge-Kutta method and using a random number generator [25] for the noise term $\xi(x, t)$.
} 


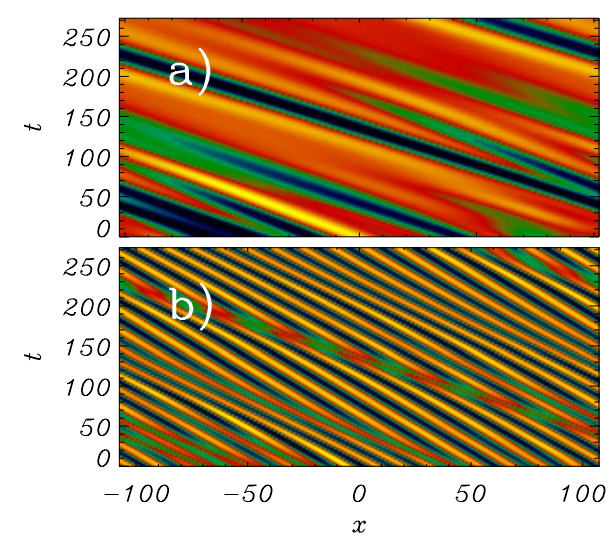

FIG. 8. (Color online) Spatiotemporal diagrams of the field intensity (a) and of real part (b) for $\delta=0.45 \pi, r=0.16, \phi=0.5 \pi, \theta$ $=0.2, \mu=0.98$, and $\Delta x=1$. Numerical simulation with noise amplitude $\epsilon=10^{-4}$ and periodic boundary conditions.

The case $\phi=\pi$ is interesting. Even if both $+k_{I}$ and $-k_{I}$ are unstable, we do not find an intensity stripe pattern above threshold. Actually, this is similar to what happens in a laser without shifted feedback, whose existence of exact traveling phase patterns is known, while intensity waves are not an exact solution [21]. The novelty here is that two waves with wave vectors $\pm k$ move with opposite velocities. In the simulations, we observe two phase patterns occupying two separate regions in the transverse dimension. These phase patterns travel apart so that on the right side we see a phase structure with positive phase and group velocities while on the left side the rolls have both velocities with negative signs.

An example is displayed in Fig. 9, representing a very peculiar noise sustained structure in which both positive and negative velocities are present. This demonstrates how nonlocality enables us to generate structures with any drift (depending on control parameters) and also to simultaneously have velocities of opposite signs in the same spontaneous structure. The symmetry of the motion in this "thread" pattern is surprising when considering the directional character

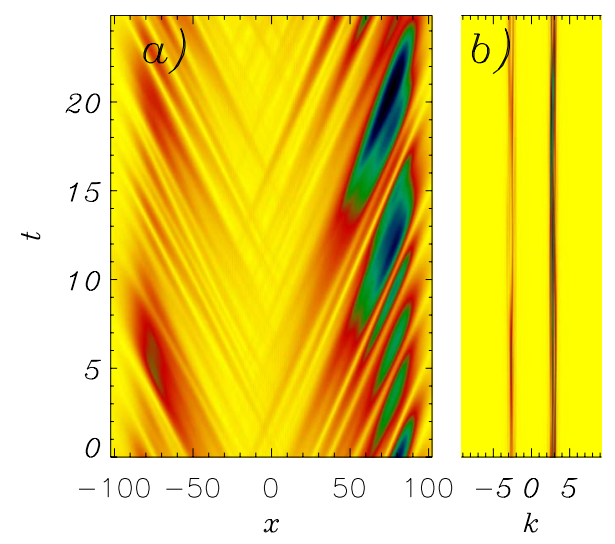

FIG. 9. (Color online) Spatiotemporal diagrams of the (a) nearfield and (b) far-field intensities. Parameters $\delta=0.49 \pi, r=0.5, \phi$ $=\pi, \theta=0.2$, and $\Delta x=1$. Simulation with white Gaussian noise of amplitude $\epsilon=10^{-3}$ and super-Gaussian pump $\mu$ with maximum value $\mu=0.98$.

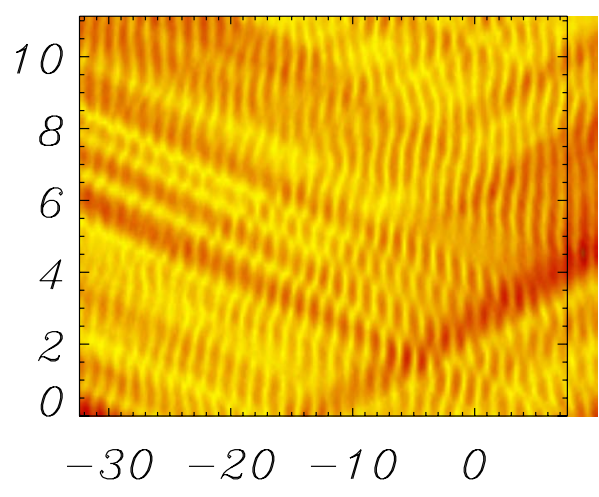

FIG. 10. (Color online) Zoom of the spatiotemporal diagrams of Fig. 9 representing a roll pattern in the intensity of the field.

of our nonlocal coupling, linking, in this case, each point with a shifted one in the positive direction. Nevertheless, a more careful observation of the pattern reveals that the patterns traveling apart have similar aspect but different intensities. The spatiotemporal diagram of the near-field intensity in Fig. 9 (left) shows a pattern with a larger intensity in the positive direction than in the negative one. Consistently, positive wave vectors are more intense as seen in far-field intensity [Fig. 9 (right)]. Note that, in presence of antiphase feedback $(\phi=\pi)$, linearly amplified perturbations are governed by a dispersion relation that is completely symmetrical in the wave number. This is a nonlinear effect and is due to a break of the reflection symmetry, as first reported in [22] for a parametric oscillator with transverse drift and then studied analytically in [23]. As it happens for the drift, also a twopoint nonlocality breaks the reflection symmetry $-x \rightarrow x$ and leads to states of the full nonlinear equation, with unbalanced intensities for far-field opposite points $|E(-k)|^{2} \neq|E(k)|^{2}$.

As waves with opposite wave vectors travel in opposite directions there is a region in which they meet after having experienced a similar amplification, traveling from opposite regions of the device transverse area. In this central region it is possible to observe partially coherent nontraveling stripe patterns also in the intensity of the field, as shown in Fig. 10, enlarging an area of the previous spatiotemporal plot. These patterns are not solutions of laser models without feedback [21]. The details of traveling fronts can be easily recognized, as superposed to these stripes.

\section{SIGNAL CONTROL}

In Ref. [14] we have proposed a method to control the spatiotemporal dynamics of localized perturbations based on off-axis feedback. Section II presents with some detail the dependence of instability thresholds and phase and group velocities on different control parameters within a linear stability analysis. Our results suggest that spatial signals can be controlled in their amplification rate, motion, or chirping, with the proper parameters choice. In particular, these signals can be controlled without changing the alignment of the device by tuning the feedback phase, suggesting the possibility to use these intense localized signals over a dark background for optical communications or computation. In this section 

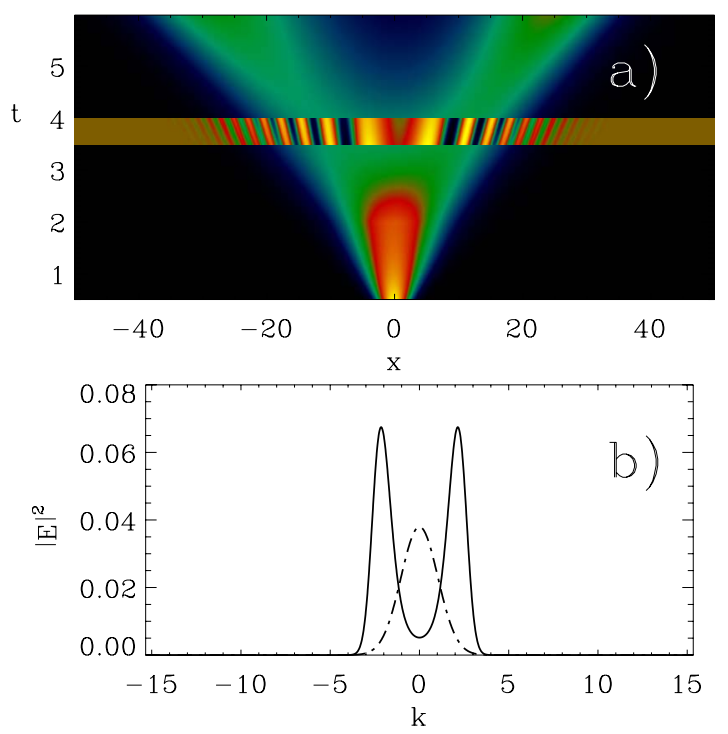

FIG. 11. (Color online) (a) Spatiotemporal diagram of the intensity of a signal under deterministic dynamics [Eq. (14)] with $\epsilon=0$. Parameters: $\delta=0.49 \pi, \theta=0.2$, and $\mu=\beta_{R}+1=1.2$. The initial perturbation is a small Gaussian and for $t<2, r=0$. The feedback is introduced only for $t>2$ and with $r=0.5, \phi=\pi$, and $\Delta x=1$. The inset for $3.5<t<4$ represents the real part of the field. (b) Far-field intensity for $t=1$ (dash-dotted line) and $t=6$ (continuous line).

we show two examples of the signal dynamics in the most peculiar case; the case of a feedback with an accumulated phase $\phi=\pi$. In this case the device works as a signal splitter, as first proposed in Ref. [14].

In Fig. 11(a) we consider a system in which an initial (Gaussian) perturbation is injected and left to evolve in absence of feedback (for time $t<2$ ) under the effects of diffraction and a small diffusion, in the absolutely unstable regime (note that no convective instability is present due to the absence of feedback and nonlocality in this first time interval). The off-axis feedback is injected at $t=2$ with a phase $\phi=\pi$, operating then the laser as a signal splitter. This implies a change in the stability and the existence of two symmetric unstable wave vectors, with opposite velocities. As a result, the signal evolves in two components propagating in opposite directions of the system. The far-field intensity represented in Fig. 11(b) shows which are the amplified wave vectors with (continuous line) and without (dash-dotted line) feedbacks. Small wave numbers are damped when the feedback beam is injected, while larger off-axis components are amplified. For our choice of parameters, when the feedback is injected, we are in the convectively unstable regime. Therefore in spite of the diffraction and spread of the two counterpropagating signals, they are eventually separated by a dark region, as the intensity locally decays.

An interesting question is the performance of this device as a signal splitter in more realistic conditions, including the presence of a source of noise. In Fig. 12(a) we show the stochastic evolution of the intensity of the field, starting from a localized Gaussian perturbation. As we are immediately above the instability threshold, in the convective regime, the noise is amplified and tends to give rise to a macroscopic incoherent structure. Nevertheless, the split of the signal in
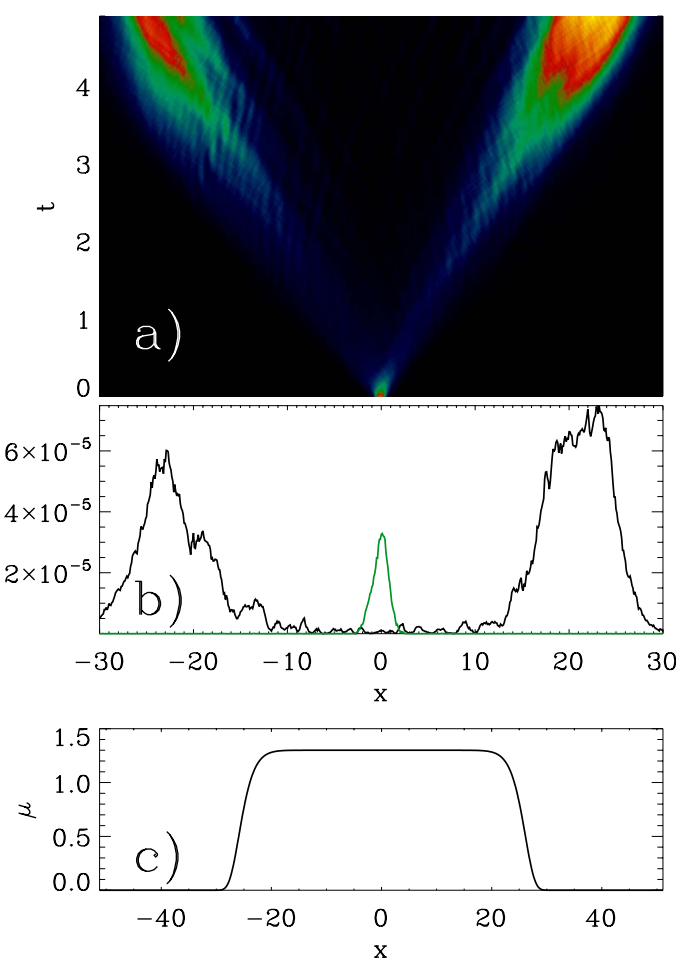

FIG. 12. (Color online) (a) Spatiotemporal diagram of the field intensity for $\delta=0.49 \pi, r=0.5, \theta=-0.5, \phi=\pi$, and $\Delta x=1$ in presence of an additive white Gaussian noise of amplitude $\epsilon=10^{-4}$. (b) Intensity profile for $t=0.2$ (green line) and split signals for $t=4.6$ (black line). (c) Pump profile $\mu(x)$ with plateau value 1.3.

two counterpropagating ones dominates, with a rather good contrast, as can be appreciated in Fig. 12(b). Features of the noise sustained structure appear as a modulation in the intensity profile.

\section{CONCLUSION}

In this paper we have given a method to study instabilities in systems with a two-point nonlocality introduced by an off-axis feedback. Using this method, we have shown that the off-axis feedback changes dramatically the dynamics of lasers with fast decaying polarization. In particular, we have found a wide range of control parameters where the laser off state is convectively unstable. In this range of parameters localized perturbations can be amplified and can have transverse group velocities positive or negative depending on the parameter values chosen; similarly, the phase velocity can be positive or negative. This remarkable tunability enables to control the direction of propagation of localized light beams and also their spatial chirping. Most importantly from the standpoint of applications, our simulations show that these properties are robust with respect to noise. While the bulk of this work has been done considering feedbacks with time delay negligible with respect to the time scales of the laser dynamics, we have shown analytically that this tunability is present also when the feedback delay time is comparable to the time scales of the dynamics. These features make possible for these systems to be considered for applications such 
as optical beam routing and multiplexing. Finally, we mention that there is a formal analogy between the variables $t, x$ considered here and the variables $z, \tau$ corresponding to the propagation length and the pulse time of pulsed lasers. From a theoretical point of view, this suggests that the effects described in this paper can be observed in a completely different context, where the tunability and chirping of spatial pulses becomes tunability and chirping of temporal pulses, provided that a two-point nonlocality can be produced by an appropriate feedback. Indeed convective instabilities have been predicted and numerically observed [24] in fiber ring lasers and result from a nonlocal term although not of the form discussed in this paper.

\section{ACKNOWLEDGMENTS}

This work was supported by the "Consejo Superior de Investigaciones Científicas" (ECuSCo under Grant No. 200850I047), by the "Ministerio de Ciencia e Innovación" (FISICOS under Grant No. FIS2007-60327), and by the Balearic Government (QULMI under Grant No. Progecib$5 \mathrm{~A})$.

\section{APPENDIX A}

In this appendix we use the rescaled variables $w=\Delta x^{2} \omega$, $t^{\prime}=t / \Delta x^{2}, q=k \Delta x$, and $x^{\prime}=x / \Delta x$ for $\Delta x \neq 0$ to give a detailed analysis of the asymptotic Green's function

$$
\lim _{t^{\prime} \rightarrow+\infty} \int_{-\infty}^{+\infty} e^{w\left(q_{I}\right) t^{\prime}+i q_{I} x^{\prime}} d q_{I} .
$$

The position of the saddles is given by the solutions of the real equation

$$
q_{I}=-\frac{\Delta x^{2} r}{2} e^{q_{I} \cot \left(q_{I}+\theta-\delta\right)} \sin \left(q_{I}+\theta-\delta\right),
$$

and $q_{R}$ depends on $q_{I}$ through the equation

$$
q_{R}=q_{I} \cot \left(q_{I}+\theta-\delta\right) .
$$

All saddle points are inside horizontal stripes in the $q$ plane, and their number varies only when two first-order saddles collide in $q=1$ or when saddles move to the singularity at infinity. Equiphase and equiamplitude lines do not depend on the additive parameter $\beta$ and are given by the equations

$$
\begin{aligned}
& \operatorname{Im}\left\{e^{i \delta} q^{2}+\Delta x^{2} r e^{q+i \theta}\right\}=W_{I}, \\
& \operatorname{Re}\left\{e^{i \delta} q^{2}+\Delta x^{2} r e^{q+i \theta}\right\}=W_{R},
\end{aligned}
$$

with $W=w-\Delta x^{2} \beta$ evaluated at any point along the path.

For $0<\delta<\pi / 2(-\pi / 2<\delta<0), W_{I}$ has an absolute maximum (minimum), $W_{t}$, on the imaginary axis $q_{I}$, and therefore, equiphase passing through saddles with $W_{I}>W_{t}\left(W_{I}\right.$ $\left.<W_{t}\right)$ cannot intersect the axis $q_{I}$. For $W_{I} \leq W_{t}\left(W_{I} \geq W_{t}\right)$ the number of intersections is finite. The asymptotes of the equiphase lines for $q_{R}>0$ are $q_{I}=2 n \pi-\theta^{+}$with $w_{R} \rightarrow+\infty$, $q_{I}=(2 n+1) \pi-\theta^{ \pm}$with $w_{R} \rightarrow-\infty$, where the upper signs apply to the case $0<\delta<\pi / 2$ and the lower signs to the case $-\pi / 2<\delta<0$; for $q_{R}<0$ the asymptotes are $\cos \delta / 2 q_{R}$ $-\sin \delta / 2 q_{I}=0$ with $w_{R} \rightarrow \pm \infty, \sin \delta / 2 q_{R}-\cos \delta / 2 q_{I}=0$ with $w_{R} \rightarrow \mp \infty$. The last two asymptotes are approached from inside for $W_{I} \delta>0$ and from outside for $W_{I} \delta<0$. The fact that equiphase lines from saddle points with different values of $W_{I}$ cannot intersect one another together with the dependence of Eq. (A4) on $W_{I}$ at infinity allows us to identify the asymptotes of the equiphase lines for each saddle point.

As mentioned in the paper, we form a closed integration path with steepest-descent paths and a finite segment $\left[q_{I_{m}}, q_{I_{M}}\right]$ on the imaginary axis, with $q_{I_{m}}, q_{I_{M}}$, such that $w_{R}\left(0, q_{I}\right)<0$ for $q_{I} \leq q_{I_{m}}, q_{I} \geq q_{I_{M}}$. We take the steepestdescent paths passing from $q_{I_{m}}, q_{I_{M}}$, i.e., equiphase lines in the direction of $w_{R} \rightarrow-\infty$; these have the same asymptotes of the steepest descents from either one or two saddles. In the first case, the steepest-descent paths from $q_{I_{m}}, q_{I_{M}}$ and the saddle point close integration path. This is the case of Figs. 4 and 5. When the steepest-descent paths from $q_{I_{m}}, q_{I_{M}}$ have the same asymptotes of the steepest descents from two saddles, $s_{m}, s_{M}$, as in Fig. 6, we need to check how the integration path is closed. For the control parameters used in this paper, the integration path is closed by steepest-descent paths from $q_{I_{m}}, q_{I_{M}}, s_{m}$, and $s_{M}$; in general, however, one may need steepest-descent paths from other saddle points in order to connect $s_{m}$ and $s_{M}$. These are the saddles in the set

$$
P_{\delta, r, \theta}=\left\{s_{i} \mid m \leq i \leq M, W_{I}\left(s_{i}\right)=\max _{M \geq j \geq i}\left[W_{I}\left(s_{j}\right) \mid W_{I}\left(s_{i}\right)<W^{t}\right]\right\}
$$

for $\delta>0$, and in the set

$$
P_{\delta, r, \theta}=\left\{s_{i} \mid m \leq i \leq M, W_{I}\left(s_{i}\right)=\min _{m \leq j \leq i}\left[W_{I}\left(s_{j}\right) \mid W_{I}\left(s_{i}\right)>W^{t}\right]\right\}
$$

for $\delta<0$. The integration contour is closed by the equiphase lines of $q_{I_{m}}, q_{I_{M}}$ and the steepest descents of the saddles in $P_{\delta, r, \theta}$. As the contribution, $I\left(q_{I_{m}}\right)$, to the Green's function from the integration along the equiphase lines from $q_{I_{m}}$ is

$$
I\left(q_{I_{m}}\right) \sim \frac{e^{w\left(q_{I_{m}}\right) t^{\prime}}}{t \partial_{q_{I}} w_{r}\left(q_{I_{m}}\right)},
$$

and an analogous formula holds also for $q_{I_{M}}$, we see that the threshold for absolute instability is

$$
w_{M}=\max \left\{w\left(s_{i}\right) \mid s_{i} \in P_{\delta, r, \theta}\right\}=0 .
$$

For $w_{R}\left(0, q_{I}\right)>0$ for some $q_{I}$, the instability is absolute when $w_{M}>0$ and convective when $w_{M}<0$.

\section{APPENDIX B}

We show in this appendix that phase and group velocity can be tuned and have the same or opposite sign even when $\Delta t$ cannot be neglected in the slowly varying amplitude. When the time delay $\Delta t$ is comparable to the time scale of the slowly varying envelope, the dispersion relation is 


$$
D\left(\omega, k_{I}\right)=-\omega+\beta-e^{i \delta} k_{I}^{2}+r e^{-\omega \Delta t+i\left(\theta+k_{I} \Delta x\right)}=0,
$$

where the term $e^{-\omega \Delta t}$ takes into account the variation in the field amplitude during the feedback loop. Equation (B1) implicitly defines $\omega$ as a function of $k_{I}$. From

$$
\partial_{k_{I}} \omega=-\frac{\partial_{k_{I}} D}{\partial_{\omega} D}=\frac{-2 e^{i \delta} k_{I}+i \Delta x r e^{-\omega \Delta t+i\left(\theta+k_{I} \Delta x\right)}}{1+\Delta t r e^{-\omega \Delta t+i\left(\theta+k_{I} \Delta x\right)}},
$$

which is valid for $\partial_{\omega} D \neq 0$, it is possible to infer that there are manifolds in the control parameters where $\partial_{k_{I}} \omega=0$. Of these, those that satisfy the condition $\partial_{k_{I}^{2}}^{2} \omega_{R}<0$ correspond to pulses with null transverse group velocity. On these manifolds, the phase velocity is given by Eq. (8) and is in general non-null. By continuity, this means that there are regions of the control parameter space where the group and the phase velocities have opposite signs and regions in which they have the same sign. This shows that the tunability of the velocities discussed in the paper is present also when the time delay of the feedback is comparable to the time scales of the dynamics.
[1] Foundations of Systems Biology, edited by H. Kitano (MIT, Boston, 2001)

[2] G. F. Franklin, J. Powell, A. Emami-Naeini, Feedback Control of Dynamic Systems (Prentice-Hall, Englewood Cliffs, NJ, 2002).

[3] B. Saleh and M. Teich, Fundamentals of Photonics (Wiley, New York, 1991).

[4] F. Arecchi, S. Boccaletti, and P. Ramazza, Phys. Rep. 318, 1 (1999).

[5] R. Lang and K. Kobayashi, IEEE J. Quantum Electron. 16, 347 (1980).

[6] A. Argyris, D. Syvridis, L. Larger, V. Annovazzi-Lodi, P. Colet, I. Fischer, J. Garcia-Ojalvo, C. R. Mirasso, L. Pesquera, K. A. Shore, Nature (London) 438, 343 (2005).

[7] P. Ramazza, S. Ducci, and F. Arecchi, Phys. Rev. Lett. 81, 4128 (1998).

[8] S. Rankin, E. Yao, and F. Papoff, Phys. Rev. A 68, 013821 (2003).

[9] L. Pastur, U. Bortolozzo, and P. Ramazza, Phys. Rev. E 69, 016210 (2004).

[10] J. P. Seipenbusch, T. Ackemann, B. B. B. Schäpers, and W. Lange, Phys. Rev. A 56, R4401 (1997).

[11] E. Louvergneaux, C. Szwaj, G. Agez, P. Glorieux, M. Taki, Phys. Rev. Lett. 92, 043901 (2004).

[12] G. Agez, P. Glorieux, M. Taki, and E. Louvergneaux, Phys. Rev. A 74, 043814 (2006).
[13] F. Papoff and R. Zambrini, Phys. Rev. Lett. 94, 243903 (2005)

[14] R. Zambrini and F. Papoff, Phys. Rev. Lett. 99, 063907 (2007).

[15] P. Coullet, L. Gill, and F. Rocca, Opt. Commun. 73, 403 (1989).

[16] P. Kockaert, P. Tassin, G. V. der Sande, I. Veretennicoff, and M. Tlidi, Phys. Rev. A 74, 033822 (2006).

[17] A. Dunlop, W. Firth, and E. Wright, Opt. Commun. 138, 211 (1997).

[18] R. Zambrini and F. Papoff, Phys. Rev. E 73, 016611 (2006).

[19] F. Papoff, G. D’Alessandro, and G.-L. Oppo, Phys. Rev. Lett. 100, 123905 (2008).

[20] R. Zambrini, S. Barnett, P. Colet, and M. S. Miguel, Phys. Rev. A 65, 023813 (2002).

[21] P. K. Jakobsen, J. Lega, Q. Feng, M. Staley, J. V. Moloney, and A. C. Newell, Phys. Rev. A 49, 4189 (1994).

[22] R. Zambrini and M. S. Miguel, Phys. Rev. A 66, 023807 (2002).

[23] R. Zambrini, M. S. Miguel, C. Durniak, and M. Taki, Phys. Rev. E 72, 025603(R) (2005).

[24] A. Mussot, E. Louvergneaux, N. Akhmediev, F. Reynaud, L. Delage, and M. Taki, Phys. Rev. Lett. 101, 113904 (2008).

[25] R. Toral and A. Chakrabarti, Comput. Phys. Commun. 74, 327 (1993). 